æmia and swelling of a valve sufficient to give rise to a deposit of fibrin, and we can imagine such deposit being washed off and lodging in one of the branches of the Sylvian artery, giving us a "brachial monoplegia" and leaving the heart practically sound. I say we can imagine this, and there is no harm in doing so if we distinguish between imaginings and proved facts. The case presents difficulties from every point of view, but these speculations are by no means idle because they have a must important bearing on the prognosis, as I have explained. The results of treatment in the case under consideration have been most excellent up to a certain point. When we have to do with a limb which is stiffened, wasted, and beyond, so to say, the reach of the will, our chief aim should be to replace voluntary exercise by every means which is available, and passive movements, rubbing, artificial warmth, and electricity will be found of service. With a limb in the condition of the one we are considering the worse course to pursue is to leave it alone, for it will inevitably result that the disused joints will get, so to say, glued up, and the limb will soon assume a position of stiffened contracture, as has happened in the case under consideration. Every joint must be methodically worked in every direction so as to maintain absolute freedom of movement. I do not think that, unless the stiffness be very excessive, much good is got by forcible movement under an anæsthetic. If, after such forcible movement, daily exercises and passive movements be not persisted in the joint will soon be glued up as tightly as before, and since the daily exercises are in any case necessary it is generally advisable to trust to these alone, and to a moderately forcible and judicious coaxing of the stiffened limb, rather than a too forcible extension. This has been done in the present case with admirable zeal by my clinical clerk, and with the best results, as the stiffness and creaking of the joints have disappeared, and the freedom of movement of shoulder, elbow, wrist, and finger-joints has been greatly increased. The next point is to improve the nutrition of the limb, and this is done by causing a "determination of blood" to it. The passive movements of the joints have done much to quicken the blood stream. In fixed limbs the venous current does not receive the help of movement, whether articular or muscular, and the passive movements of the joints and the kneading of the muscles do much to counteract the evils of this deficiency. Artificial warmth is all-important in improving the nutrition, and to warmth should be added a stimulation of the skin sufficient to produce a thorough redness of the surface. Such a limb should be kept in a flannel sleeve, and the hand should be covered with a warm glove so as to run no risk of its getting chilled. Warm bathing is also of great use, and when the trouble is strictly local, as in this case, a soaking of the limb in hot water is of service, the soaking being followed by passive movements and massage. It is of some use, probably, to add salt to the water, as thereby its stimulating effect upon the surface is increased. Had our patient been rich he would very probably have been sent to Bath, Aix-lesBains, or some similar place, where the machinery for bathing and rubbing and douching has been elaborated to the utmost; but I would have you observe that it is possible to do a great deal by similar methods employed with a will at home.

Electricity is of great service in these cases. If the irritability of the muscles is lessened the application of faradisation is often very useful, and it should be employed until the irritability reaches the normal. There has been no noticeable diminution of irritability in this case, and none of the muscles give the "degenerative reactions," so that the good effects of faradisation have not been as noticeable as sometimes is the case. The good effects of faradisation are, in cases like this, often very marked at first. By its use we succeed in "stirring up" the disused muscles, and greatly increased freedom of movement is the result. The effect of the first application is often very marked, occasionally it is astounding, while subsequent applications produce hardly any noticeable effect. The continuous current is also extremely serviceable. Large sponge rheophores should be used, and these should be moistened with salt-and-water made as hot as it can be borne. I know of nothing equal to this as an effectual skin reddener, and it is undoubtedly to its power in this direction-the causing a "determination of blood" to the limb-that much of the utility of the continuous current is due. Cases of this class are often very chronic. Chronic rheumatic affections are very prone to last (with considerable exacerbations and remissions) for a lifetime, and contractures following upon cerebral lesions will certainly do so. The patient's discom. forts are very much lessened if we can maintain the nutri. tion and the suppleness of the limb. This is done by the methods I have indicated, and it is very advisable to use one method at a time and not to exhaust your resources alk at once. Human nature loves a change, and will have a variety, and far be it from me to say that change and variety are not of solid use. We all strive to get, and all enjoy, a change in the matter of diet, and that much in excess of what the physiologists tell us is necessary. So the chronic invalid, the nutrition of whose paralysed or rheumatic limb has to be artificially maintained, will have a change of method. It is, therefore, advisable to use one method at a time. Massage, hot bathing, electricity, liniments, \&c., are all means of maintaining nutrition, and when the patient has tired of one method we may try a second. As soon as the limb is left to itself it will stiffen and get glued up and fixed. The great use of liniments in such cases is found in the fact that they are all so to say vehicles for friction. The only thing to bear in mind about them is that they should be oily and not too stimulating. Great care must be taken that no excegs of zeal in the matter of rubbing produces any abrasion of the skin, and it is in order to avoid the risk of skin abrasion that I recommend the use of oily liniments which serve to lubricate the surface. It is needless to say that the patient should be encouraged to use the damaged limb as much aq possible, and the exercises prescribed will of course depend upon the amount of voluntary power. In order to keep the shoulder- and elbow-joints supple it is a good plan to get the patient to work the limb by means of an elastic band with a stirrup handle, which must be hung to the ceiling or the wall of his room. With methods like those I have been describing our patient has improved very rapicily indeed up to a certain point. The joints move easily, and the contraction of the arm and fingers has disappeared. His power of using the limb has not kept pace with the greatly improved local condition, and it is this fact which strengthens and confirms me in the diagnosis of a central cerebral lesion in addition to his local rheumatism. By local measures we may succeed, as we have done, in loosening stiffened joints and in improving local nutrition. There is, however, the scar of an old injury in the area for the arm in the motor region of the cortex cerebri on the right side. This is not to be reached by our rubbings, frictions, and clectricity, and although the local improvement has been immense, there has not been and never will be any commensurate improvement in the voluntary use of the limb. It is easy, therefore, to see the great importance of diagnosis and its influence on prognosis.

\section{ON TOTAL EXTIRPATION OF THE UTERUS BY THE VAGINA. ${ }^{1}$}

BY JOHN WILLIAMS, M.D., F.R.C.P.

CANCER of the uterus per se is an indication for total extirpation of the organ, and yet all such cases are not fit subjects for the operation. There are several consideras tions which show this. Total extirpation is an opera. tion which should be undertaken with a view to radical cure only. It is of too grave and mutilating a character to be adopted as a merely palliative measure. All cases, therefore, in which it is clear that the disease cannot be entirely rem ved are unsuitable for the procedure, so that in those cases in which the growth bes invaded neighbouring organs--the bladder, rectum, vasina, broad ligaments, or intestines-the operation is out of the ques. tion. This one consideration, unfortunately, sweeps off as unfit subjects for total extirpation the great majority of the cases of cancer of the uterus which present themselves at our hospitals. We are consulted when the disease is so far advanced as to render radical measures impossibie. With regard to the remaining cases, it is necessary to enter into some particulars-particulars of diagnosis, seat of origin of

1 Being the address opening the discussion on the surject at the International Medical Congress, Berlin. 
the disease, degree of extension of the growth, the presence or absence of secondary growths, the possibility of the removal of the organ by the vagina, and the condition of the general health. Cancer may originate in the uterus in one of three places-in the body, the cervix, or the vaginal portion of the cervix. The history and character of the growth differ according to the part which is first attacked, and from them certain indications for operative interference may be obtained.

In cancer of the body of the uterus the operation is indicated, inasmuch as no other measure is capable of removing the disease. Yet all cases, even of this form of cancer, are not fit subjects for the surgeon's skill. The operation should be undertaken only when the whole of the diseased tissue can be removed; in the early stages, therefore, before the parametric tissues and the peritoneum have been invaded or infected. This involves much more than mobility, and even free mobility, of the uterus, and I sball refer to this again when speaking of the diagnosis of the conditions in which operative interference is indicated. Adhesions between the uterus and neighbouring organs may be present. They may be cancerous or simply inflammatory. When they are cancerous the disease will have attained an advanced stage, and there will be other evidence of this : in the broad ligaments in the form of thickening or glandular swellings, and in the cavity of the uterus which may have been converted into a foul abscess. Occa. sionally, however, cancerous adhesions may have formed between the uterus and the intestine without giving direct evidence of their presence. The ditficulty of distinguishing between adhesions due to cancer from those due to simple inflammation is great, and I know of no way of overcoming it except in some cases in which adhesions were known to exist before the development of the malignant disease. For the operation of total extirpation by the vagina which is now being discussed, the volume of the uterus gives important indications. In cancer of the body the organ varies much in size. It may be litule bigger than the uterus frequently met with in a multiparous woman, or it may be so large as to project above the pelvic brim and reach to the umbilicus. When the increase in volume is such that the fundus does not rise above the pelvic brim, the organ can be removed by the vagina, but when the organ is so large as to reach the umbilicus total extirpation by the ragina becomes impossible. What, then, are the exact limits of size which contraindicate extirpatio uteri vaginalis?

It is with regard to the indications for the operation in cancer of the cervix and of the vaginal portion that opinions differ most widely. Is the operation indicated in these forms of the disease? and, if indicated, under wha circumstances? Much has been said and written upon this part of the subject, and diverse opinions held and expressed. The true answer to these questions must depend upon an elaborate inquiry of two kinds-anatomical and clinical. The anatomical and clinical history of cancer of these parts must be known before a final reply to these questions is possible. Anatomical research must be conducted under two conditions: (l) When the disease has been allowed to run its course without surgical interference, and (2) when the minor operation-supra-vaginal amputation of the cervix with or without the help of the ferrum candens-has been resorted to. These methods will complement each other, and both will supply us with valuable indications. But the first inquiry is, What is the anatomical history of cancer of the cervix when left to itself? The starting-point of cancer of the cervix is usually the glands, perhaps the deeper parts of the glands. This is proved by those cases in which, over a mass of cancer lodged in the substance of the cervix, the epithelium lining the cervical canal remains intact. This is no uncommon occurrence. Again, the seat of origin of the disease may be a point in the upper half of the cervix near the inner orifice, or a point in the lower half near the outer orifice. The lower starting-point appears to be the most favoured, while the cases in which the disease begins in the upper half appear to be somewhat rare. Does cancer ever begin in the superficial epithelium lining the cervical canal? It probably does, while it is certain that it may commence in the bottom of the spaces between the papillw The course of the disease, the direction of its growth, when it owns the latter origin, have not been worked out. What is the direction of its growth when it commences in the glands of the cervix? Does it tend to invade the body or the circum-cervical tissues? When the body of the uterus is affected, in what manner is the in. vasion brought about? Is it by direct extension from the cervix, or by secondary deposit, or by a synchronous de. velopment of cancer in the body and the cervix, or is it indirectly through the medium of the circum.cervical tissues? And, finally, at what period of the disease does the body become affected? Is it before or after infection of the parametric tissue has taken place?

All these questions have an important bearing upon the indications for total extirpation. The usual tendency of this form of cancer, be its starting-point near the inner or near the outer orifice, is to involve the whole thickness and length of the cervix, and to pass through the cervix into the circum-cervical tissues, but respecting for a time the inner orifice. When it begins near the outer orifice, it spreads outwards and upwards respecting the squamous epithelium covering the vaginal portion, but hastening into the connective tissue around the cervix. When it begins near the inner orifice its tendency is to grow ontwards towards the connective tissue and downwards, but not upwards. The connective tissue is invaded before the body is attacked and before the epithelium covering the vaginal portion is destroyed and ulcẻration takes place. That the body becomes affected at some period of the disease is, how. ever, sufficiently evident. Abundant proof of it is found in every hospital museum. In these museum specimens, how. ever, is it not the case that the circum-cervical tissues are everywhere cancerous and breaking down, that the parametric tissues in the broad ligaments and the outer surface of the uterus, almost as far as the insertion of the Fallopian tubes, are involved, that the cervix and the circum-cervical tissues have largely sloughed or ulcerated away, and that the lower portion of the body dips into a sloughing cavity formed by the destruction of not the cervix only, but also of the parts around? This appears to show that in the later stages of the disease extension into the body takes place directly, but it shows further that this extension is preceded by indirect extension to the outer surface of the body through the medium of the connective tissue around. The outer surface of the body-the sides especially-is affected earlier and to a higher point than the inner. Are there exceptions to this general conduct of cancer of the cervix? There appear to be rare instances in which the disease extends superficially along the epithelium into the body, and this possibly occurs in those cases in which the disease begins in the superficial epithelium of the cervix.

There are other cases in which cancerous masses or nodules are found in the inner superficial layers of the body, together with an early stage of cancer of the cervix. These nodules are isolated, and cannot be due to direct extension from the cervix. Two views may be held with regard to them. One is that they are secondary deposits the primary disease being that in the cervix. It is ditficul to understand how secondary deposits can be formed in that situation. The other, and the view which appears more probable, is that these nodules are new formations, inde pendent of the cervical disease. They are not common. But what is the anatomical history of the disease after supra-vaginal amputation of the cervix? What is the seat of recurrence, and what is the direction of its growth. Recurrence takes place in the great majority of cases within a limited time. Its usual seat, however, is not the stump of the uterus, but the connective tissue. The cancer grows rapidly in this tissue, and spreads upwards into the broad ligaments, and downwards between the bladder and the vagina, and in the recto-vaginal septum, leaving the uterine stump intact. Even after death the stump may be only slightly affected, and that only on its surface, showing that the attack on the stump is the last effort of the disease at extension. It is hardly necessary to mention that after total extirpation of the uterus the disease recurs in the parametric tissues. Of course it may recur in other organs, such as bones, glands, and viscera Cancer of the raginal portion begins in the squamous covering of that organ and is a squamous epithelioma. It tendency is not to spread deeply but superficially along the portio vaginalis to the vaginal walls. Along these walls it spreads superficially towards the vulva. In its later stages it spreads deeper into the connective tissue, but in its earlier stages, when a radical cure may be effected, its depth is but slight, even less than half an inch. There are some exceptions to this, in which the disease sends pro cesses deeply into the cervix, into its inner layers; these soon ulcerate and split the cervix into two rings; the inner 
subsequently sloughs off and a great conical cavity is formed in the situation of the os uteri surrounded by a layer of cervical tissue. After amputation of the cervix the disease recurs in the epithelium at the edge of the cicatrix and is quite superficial, and not in the stump.

The clinical inquiry turns upon the frequency of recurrence, the time which has elapsed before recurrence after total extirpation and supra-vaginal amputation, and the use of the actual cautery, and upon the comparative danger of the two operations. What are the results as to permanent cure after total extirpation? Are they better than those obtained by supra-vaginal amputation of the cervix? Here our present statistics are insufficient to warrant a positive conclusion. This is accounted for (1) by the difficulty of following cases for a sufficiently long period after operation, and (2) by the shortness of the time within which the operation has come to be practised. At one time the statistics of supra-vaginal amputation appeared to be better than those of total extirpation. This was due to the fact that supra-vaginal amputation was adopted in the less and total extirpation in the more advanced cases upon what appears now to be an erroneous view of the progress of cancer, for it is not reasonable to suppose that the minor operation can be more effectual in eradicating the disease than the major. The brilliant results obtained after total extirpation by our confrères in Germany show that in their hands the danger of this operation has been reduced to comparative insignificance. From over 70 per cent., the mortality was first reduced to 28 , then under 20, then under 10, and now, in some instances, as low as 5 per cent. This is, perhaps, the greatest of the many marvels of modern surgery. At the same time, the question must be asked, Does this mortality exceed the mortality of supra-vaginal amputation of the cervix when performed with the same care? Again statistics fail us. The statistics of partial amputation of six years ago cannot, in justice, be compared with those of total extirpation of to-day. One other question should be asked in this connexion, that is, What are the effects of the different degrees of mutilation caused by the two operations?-a question which has as yet not been fully investigated.

The bearing on practice of the anatomical and clinical considerations which have been mentioned are generally admitted. We may, however, hold certain views with regard to the indications for total extirpation, and be unable to carry them out. How are we to recognise these anatomical conditions during life ? The diagnosis of cancer in its early stages, be it in the body or in the cervix, is replete with difficulties. Clinical examination of the most complete character does not speak with certainty, and while removal of a portion for examination with the microscope may remove all doubt, yet in many cases even this fails. What shall we say, then, of the diagnosis of extension of the disease into the cellular tissue? This is still more difficult, often impossible, as is proved by the frequency of recurrence in the cicatrix. When the disease begins in the vaginal portion the superficial direction of its growth favours the diagnosis. Nothing intervenes between the examining finger and the disease, consequently the recognition of the invasion of the vaginal wall is comparatively easy. Far dufferent is it when cancer begins in the cervix or in the body. The nearer to the external orifice is the seat of the growth the less difficult will be the discovery of extension into the cellular tissue, the further from that point the more difficult. Indeed, only when the circum-eervical or parametric tissue is coarsely indurated is the discovery likely to be made. Immobility of the uterus, marked induration of the circum-cervical tissue, the broad ligaments or the sacrouterine ligaments, are coarse signs easily recognised which contraindicate the operation; but mobility of the organ does not necessarily indicate surgical interference. It is when the organ is mobile that the difficulty of diagnosis arises, and the difficulty comes from the fact that a thick layer of tissue intervenes between the examining finger and the diseased grow th. The higher the disease begins the thicker is this intervening layer and the greater the difficulty of recognising the real condition present. In cases in which the whole thickness of the uterine wall is diseased, the cellular tissue is already invaded, but we labour under the disadvantage of possessing no clinical means of detecting it. The best and only sign of it, perhaps, is a bulging or thickening, together with marked bardness of the uterine wall at th part affected. The eurrounding tisscies may appear to glide over it as in health, and during the operatic $n$ no indication is met with of disease of the cellular tissue. There is no difficulty in separating the diseased neck from the parametrium, and yet in these cases the parametrium is cancerous, and hence the frequent recurrences. Is it possible to recognise a thin layer of healthy uterine tissue over a diseased part when the disease is situated high up in the cervix? I believe this is sometimes possible, and it is in cases where this is found that a radical cure may be expected. In cancer of the body I believe it to be impossible to recognise such early invasions of the parametriumat least $I$ have not met with a case in which $I$ have been able to do so.

Our aim should be to recognise this transition stage, to distinguish the cases in which cancer is limited to the uterine tissues from those in which it has passed just beyond them, where there is no appreciable induration of the parametric tissues, and in which nevertheless recurrence after operation is certain. Both vaginal and rectal examinations, with theaid of anæsthesia, should be resorted to with a view of deciding this point, and also of discovering glandular enlargements in the pelvis. Glandular enlargements are sometimessituated at too great a distance from the uterus to be discovered, while the intervening tissue is healthy, and months after the operation become manifest by their growth, when the cicatrix, together with the remains of the broad ligaments, remain free from disease. When it is found that the whole thickness of the uterine wall or of -any part of it in the cervix or in the sides of the body where the broad ligaments are attached is involved, should total extirpation be resorted to? or does the operation undertaken under such conditions offer any hope of radical cure?

In opening this discussion I have not attempted to enter into details, but confined myself to suggesting the general principles which should guide us in the formation of correct views upon the subject, and these suggestions have been largely thrown into the form of questions to which, as yet, no complete and satisfactory answers have been made.

\section{THE MALARIAL FEVER OF MAURITIUS;} ITS MICROBIC ORIGIN AND ITS COMPLICATIONS.

By D. E. ANDERSON, M.D. PARIs, B.A., B.SC. LoND。

DURING a visit which I paid last summer to the Island of Mauritius I was enabled, through the kindness of the medical officers in charge of the hospitals of Port Louis, to obtain much interesting information concerning the peculiar type of malarial fever which for the last twenty years has been doing so much havoc in that beautiful island. Until the year 1866, Mauritius was considered to be one of the healthiest resorts in the Southern hemisphere. It had a flourishing commerce, bringing in as great a revenue as that enjoyed by England in the reign of Elizabeth. It had a general population of 120,000 souls, and an Indian popula tion of 200,000 immigrants. To-day, however, its commere is less flourishing, and the ravages of fever have reduced the general population down to less than 100,000; and although the Indian population has increased to over 218,000 , this is simply due to the more extensive scale on which immigration from India has been conducted during the last few years.

The origin of malarial fever in Mauritius is even now a question on which there is much difference of opinion. Some epidemiologists still uphold Colin's telluric effluvial theory, and argue that for ages past the malaria has lain dormant in the soil ; others, with greater show of reason, maintain that until the arrival of the emigrant ship the Spunky from India, with malarial fever on board, in 1865, this fever was not known in Mauritius, and that the germ thus introduced found the island at that time in a condition most favourable for its propagation, for the soil had been recently turned up for the railway and gas works, and two serious inundations in 1862 and 1865 , followed by pro. longed droughts, had not only filled the cellars and gardens in the lower parts of Port Louis with mud, but had also created all along the course of the rivers small stagnant pools, a condition of things not improved by the cutting down of the forest trees on the watershed of the island, which was being converted into sugar-cane fields. 